\title{
Ingestão de cálcio e densidade mineral óssea em mulheres adultas intolerantes à lactose
}

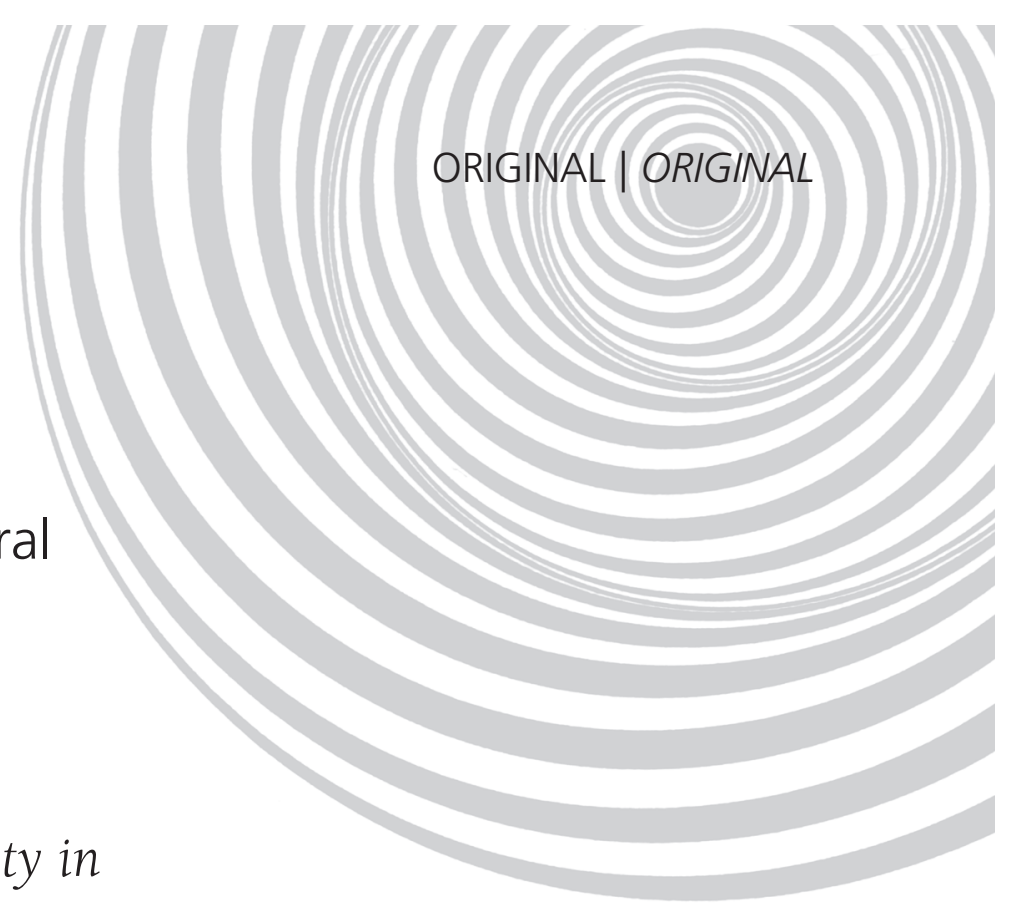

\section{Calcium intake and bone mineral density in adult women with lactose intolerance}

Najoua Adriana SALOMÃO'

Thays de Ataide e SILVA'

Amandio Aristides Rihan GERALDES'

Adriano Eduardo LIMA-SILVA'

RE S U M O

\section{Objetivo}

Comparar a ingestão de cálcio e a densidade mineral óssea entre mulheres adultas portadoras de intolerância à lactose e suas congêneres não portadoras da doença.

\section{Métodos}

A amostra foi composta por 60 mulheres jovens adultas (de 20 a 40 anos), separadas em dois grupos: 30 diagnosticadas com intolerância à lactose e 30 saudáveis. A ingestão de cálcio foi avaliada por três autorregistros alimentares, e a densidade mineral óssea do colo do fêmur por absortometria radiológica de dupla energia.

\section{Resultados}

As densidades minerais ósseas do colo do fêmur ( $M=0,86, D P=0,13 \mathrm{~g} / \mathrm{cm}^{2}$ versus $\left.M=0,77, D P=0,12 \mathrm{~g} / \mathrm{cm}^{2}\right)$ e do fêmur total $\left(M=1,14, D P=0,14 \mathrm{~g} / \mathrm{cm}^{2}\right.$ versus $\left.M=1,06, D P=0,12 \mathrm{~g} / \mathrm{cm}^{2}\right)$ foram menores $(p<0,05)$ para as portadoras de intolerância à lactose do que para o grupo-controle, mas não houve diferença significativa na densidade mineral óssea para corpo inteiro $\left(M=1,14, D P=0,15 \mathrm{~g} / \mathrm{cm}^{2}\right.$ versus $\left.M=1,08, D P=0,09 \mathrm{~g} / \mathrm{cm}^{2}, p>0,05\right)$. Além disso, a ingestão de cálcio foi menor para intolerantes à lactose do que para o grupo-controle $(M=250,5$, $\mathrm{DP}=111,7 \mathrm{mg} / \mathrm{dia}^{-1}$ vs $\left.\mathrm{M}=659,7, \mathrm{DP}=316,1 \mathrm{mg} / \mathrm{dia}^{-1}, p<0,05\right)$.

\section{Conclusão}

Os resultados do presente estudo sugerem que o aparecimento de sintomas de intolerância pode influenciar a massa óssea devido a uma redução da ingestão de cálcio.

Termos de indexação: Lactose. Leite. Osso. Sindrome de malabsorção.

\footnotetext{
$\overline{1}$ Universidade Federal de Alagoas, Faculdade de Nutrição. Av. Lourival Melo Mota, s/n., Tabuleiro do Martins, 57072-970, Maceió, AL, Brasil. Correspondência para/Correspondence to: AE LIMA-SILVA. E-mail: <adrianosilva@usp.br>.
} 
588 | NA SALOMÃO et al.

\section{A B S T R A C T}

\section{Objective}

This study compared the calcium intake and bone mineral density of adult women with lactose intolerance with those of their counterparts without the condition.

\section{Methods}

Sixty adult women aged 20 to 40 years were divided into two groups: 30 diagnosed with lactose intolerance and 30 without the condition. Calcium intake was assessed by three 24-hour recalls and bone mineral density of the femur was determined by dual energy $x$-ray absorptiometry.

\section{Results}

The bone mineral density of the femoral neck $\left(M=0.86, S D=0.13 \mathrm{~g} / \mathrm{cm}^{2}\right.$ versus $\left.M=0.77, S D=0.12 \mathrm{~g} / \mathrm{cm}^{2}\right)$ and femoral total $\left(M=1.14, S D=0.14 \mathrm{~g} / \mathrm{cm}^{2}\right.$ versus $\left.M=1.06, S D=0.12 \mathrm{~g} / \mathrm{cm}^{2}\right)$ were lower $(p<0.05)$ for the lactose-intolerant group than for the control, but there was no significant difference for the bone density of the entire body $\left(M=1.14, S D=0.15 \mathrm{~g} / \mathrm{cm}^{2}\right.$ versus $\left.M=1.08, S D=0.09 \mathrm{~g} / \mathrm{cm}^{2}, p>0.05\right)$. Also, calcium intake was lower for the lactose intolerant than for the control group $\left(M=250.5, S D=111.7 \mathrm{mg}\right.$ day $^{-1}$ versus $M=659.7$, $S D=316.1 \mathrm{mg} /$ day $\left.^{-1}, p<0.05\right)$.

\section{Conclusion}

The results of this study suggest that the onset of intolerance symptoms may influence bone mineral density due to low calcium intake.

Indexing terms: Lactase. Milk. Bone and bones. Malabsorption sindromes.

\section{N T R O D U ÇÃ O}

O leite é uma excelente fonte de proteína animal e o constituinte dietético mais adequado como fonte de cálcio'. Entretanto, a capacidade de digerir a lactose contida no leite depende da presença e da atividade da enzima lactase ${ }^{2,3}$, cuja atividade diminui com o passar dos anos devido a uma redução na sua quantidade no intestino, ocasionando a má-absorção da lactose ${ }^{4-6}$.

Na maior parte da população mundial, após o desmame, há um declínio gradual na atividade da lactase, um fenômeno denominado hipolactasia. O declínio nos níveis de lactase é progressivo durante a infância e a adolescência, havendo um aumento nas taxas de má-absorção de acordo com a idade7,8. Nos casos em que a má-absorção de lactose avança para o estágio de Intolerância à Lactose (IL), os indivíduos portadores apresentam uma série de reações adversas após ingestão de leite e de seus derivados: diarreia, flatulência, náusea, dor e distensão abdominal9,10. A presença dessas reações pode levar a um menor consumo de leite e de derivados e, consequentemente, a uma ingestão insuficiente de cálcio, predispondo seus portadores a maiores riscos para o desenvolvimento da osteoporose $\mathrm{e}^{10,11}$.

Nesse contexto, estudos têm sido desenvolvidos com o objetivo de comparar a ingestão de cálcio diário e a Densidade Mineral Óssea (DMO) entre mulheres saudáveis e portadoras de $\mathrm{IL}^{12-14}$. Corazza et al..$^{15}$ verificaram que a DMO e o consumo de cálcio eram menores em mulheres em pós-menopausa que apresentavam má-absorção de lactose e sintomas de IL quando comparadas àquelas que apresentavam apenas má-absorção, mas sem sintomas, ou com aquelas do grupo-controle. Da mesma forma, Matlik et al. ${ }^{16}$ verificaram que a ingestão de cálcio e a DMO eram menores em meninas de 10 a 13 anos com sintomas de IL do que nas meninas de mesma idade consideradas apenas má absorvedoras sem sintomas, ou das meninas não intolerantes. Dessa maneira, a presença do sintoma de $\mathrm{IL}$, e não a atividade isolada da enzima lactase, é que atua como fator determinante na redução no consumo de cálcio e, por consequência, na redução da DMO, o que sugere que a não digestão da lactose no organismo leve a sua não absorção por falta da hidrólise dessa molécula em seus constituintes 
glicose e galactose. O acúmulo de lactose no intestino induz à fermentação por microrganismos intestinais, o que resulta na formação de gases como metano, dióxido de carbono e hidrogênio, que são responsáveis pela flatulência, distensão e dor abdominal, sintomas característicos da IL. Além disso, a presença de lactose não absorvida no lúmen intestinal aumenta a pressão osmótica, retendo água e aumentando o trânsito intestinal, o que resulta em fezes amolecidas e diarreia, podendo levar a uma absorção comprometida de cálcio ${ }^{17}$ e à utilização do cálcio ósseo para a manutenção desse mineral no sangue e dos níveis de cálcio neural ${ }^{10}$.

O tratamento para indivíduos IL consiste basicamente da não ingestão de produtos lácteos na dieta, o que se torna um problema, pois esses alimentos são fontes primárias de cálcio, e, considerando que o corpo perde quantidades consideráveis desse mineral diariamente, torna-se necessário um suprimento constante de cálcio biodisponível para garantir a DMO. Apesar disso, não é conhecido se mulheres adultas portadoras de IL reduzem sua ingestão de cálcio. Alguns estudos longitudinais realizados com crianças até a idade adulta demonstraram que, com o passar dos anos, a ingestão de cálcio diminui. Nesses estudos, a presença de sintomas gastrointestinais levaram esses indivíduos a não consumirem leite e derivados, o que reduziu a quantidade de cálcio ingerida, deixando esses indivíduos suscetíveis à redução da $\mathrm{DMO}^{15,16}$.

Outros estudos de comportamento em relação ao consumo de leite nesses indivíduos apontam que, ao se evitar o consumo de produtos lácteos, há uma redução da DMO e uma maior incidência de fraturas ósseas ${ }^{10,15,18}$. Da mesma forma, Di Stefano et al. ${ }^{19}$ compararam a ingestão de cálcio e a DMO em indivíduos adultos ( 28 anos) saudáveis e portadores de IL. Di Stefano et al. ${ }^{19}$ concluíram que a ingestão de cálcio e a DMO da coluna lombar e do fêmur proximal eram menores no grupo de portadores de IL. Entretanto, a amostra utilizada pelos autores foi composta de homens e mulheres reunidos em um único grupo, o que impossibilita identificar o efeito isolado da IL sobre a $\mathrm{DMO}$, uma vez que o risco de diminuição da DMO é diferente entre os sexos. De fato, a redução do osso trabecular e do osso cortical com o avanço da idade é de aproximadamente $25 \%$ a $35 \%$ para as mulheres, e de apenas $5 \%$ a $15 \%$ para os homens ${ }^{20}$. As mulheres também apresentam menor absorção de cálcio pelo intestino do que os homens ${ }^{20}$ : dessa forma, o sexo feminino apresenta maior risco de desenvolver osteoporose quando comparado ao masculino ${ }^{21}$. Assim, um estudo utilizando uma amostra exclusiva de mulheres adultas com IL torna-se necessário.

O objetivo do presente estudo foi comparar a ingestão de cálcio e a DMO entre mulheres adultas portadoras de IL e suas congêneres não portadoras da doença. A hipótese do estudo é a de que a ingestão de cálcio e a DMO do grupo IL seriam menores do que as do grupo-controle.

\section{MÉ T O D O S}

\section{Amostra}

Para estabelecer o número mínimo de participantes necessários para obter um Power Effect superior a 0,80 e com um valor de alfa inferior a 0,05 , foram utilizados os dados de DMO descritos por Di Stefano et al. ${ }^{19} \mathrm{e}$ os de consumo de cálcio descritos por Honkanen et al. ${ }^{12}$. A partir do tamanho do efeito, calculado pelos valores de Média (M) e de Desvio-Padrão (DP) descritos por esses autores, e usando uma razão de alocação igual a um (grupo 1/grupo 2), constatou-se que seriam necessários sete sujeitos em cada grupo para identificar diferenças significantes relacionadas à DMO e 30 sujeitos para identificar diferenças significantes relacionadas ao cálcio. Portanto, considerando o custo operacional, optou-se por utilizar 30 sujeitos em cada grupo. Assim, a amostra foi constituída por 60 mulheres jovens adultas, na faixa etária entre 20 e 40 anos (Tabela 1), divididas em dois grupos: 30 mulheres com IL (Grupo 
Intolerante a Lactose - GIL) e 30 mulheres-controle sem IL (Grupo Controle - GC). A amostra foi formada por mulheres caucasianas (24 mulheres no GC e 25 mulheres no GIL) e negras (seis mulheres no GC e cinco mulheres no GIL). Fizeram parte da amostra apenas mulheres não fumantes e que não apresentavam outras doenças que pudessem interferir na absorção de cálcio, como doença celíaca ou alergias alimentares ${ }^{22}$. Todos os sujeitos assinaram o Termo de Consentimento Livre Esclarecido - previamente aprovado pelo comitê de ética da Universidade Federal de Alagoas (CEP/ UFAL) para pesquisas que envolvem seres humanos (n²3065.010008/2009-71, aprovado em 19 de outubro de 2009) - antes de sua inclusão na amostra. Os valores para idade, peso e estatura para cada um dos grupos são apresentados na Tabela 1.

\section{Desenho experimental}

Inicialmente, todas as voluntárias realizaram um teste de tolerância à lactose. Posteriormente, foram mensurados o peso corporal e a estatura, seguidos pela realização do exame de absorciometria de Raios-X de Dupla Energia (DXA) para verificar a DMO. Em seguida, as voluntárias responderam a um questionário feito na forma de entrevista sobre questões gerais (idade, presença de doenças que pudessem interferir na absorção de cálcio, uso de medicação e etnia) e foram orientadas a preencher três autorregistros alimentares, de modo que pelo menos um correspondesse a um final de semana. Todas as etapas realizadas no presente estudo estão descritas na Figura 1.

\section{Teste de sobrecarga de lactose e curva glicêmica}

Todas as participantes realizaram os testes no período da manhã, tendo respeitado o jejum de oito a doze horas. As voluntárias foram submetidas à coleta de sangue venoso em três momentos: a primeira dosagem em jejum (basal) e a segunda e terceira, 30 e 60 minutos após a ingestão de $50 \mathrm{~g}$ de lactose, respectivamente.

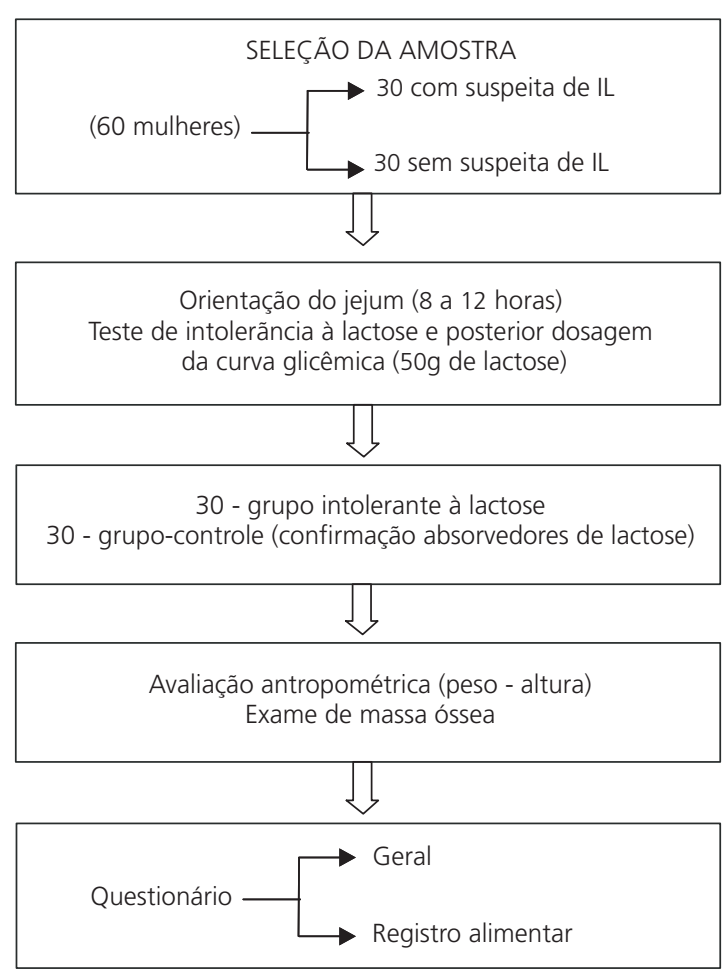

Figura 1. Desenho experimental aplicado no presente estudo. Nota: IL: Intolerante à Lactose.

Tabela 1. Valores de idade, peso corporal e estatura nos grupos-controle e no grupo intolerante à lactose. Maceió (AL), 2011.

\begin{tabular}{|c|c|c|c|c|c|c|c|c|c|}
\hline \multirow{2}{*}{ Variáveis } & \multicolumn{4}{|c|}{$\mathrm{GC}$} & \multicolumn{4}{|c|}{ GIL } & \multirow{2}{*}{$p$} \\
\hline & M & DP & Mínimo & Máximo & $M$ & DP & Mínimo & Máximo & \\
\hline Idade (anos) & 31,9 & 6,9 & 24,0 & 43,0 & 31,6 & 7,5 & 24,0 & 44,0 & 0,874 \\
\hline Peso corporal (kg) & 62,0 & 11,6 & 47,0 & 77,0 & 58,0 & 12,3 & 41,0 & 81,0 & 0,203 \\
\hline Estatura $(\mathrm{cm})$ & 160,8 & 5,8 & 150,0 & 166,0 & 160,8 & 6,6 & 51,5 & 172,0 & 0,685 \\
\hline
\end{tabular}

GC: Grupo-Controle; GIL: Grupo Intolerante à Lactose; M: Média; DP: Desvio-Padrão. 
A quantidade total de sangue venoso retirado por coleta foi de $10 \mathrm{~mL}$. O sangue foi imediatamente centrifugado a 3000 rotações por minuto durante 10 minutos a $4^{\circ} \mathrm{C}$ para separação do soro. A concentração de glicose no soro foi medida através de reações colorimétricas, em um equipamento automatizado (Olympus AU400e, Olympus Optical do Brasil Ltda, São Paulo, BR). Todas as participantes foram orientadas a registrarem as possíveis ocorrências de sintomas de intolerância experimentados durante e no período de 24 horas após o teste.

O diagnóstico de má-absorção de lactose foi feito quando a glicemia, após a ingestão da lactose, aumentou menos do que $20 \mathrm{mg} / \mathrm{dL}^{22,23}$. O diagnóstico de IL foi confirmado a partir da combinação da má-absorção de lactose com a presença de sintomas (diarreia, constipação, enxaqueca, náuseas, flatulência, distensão e dor abdominal). Somente os indivíduos diagnosticados com má-absorção e que apresentaram sintomas de desconforto intestinal, durante ou após o teste, foram alocados para o grupo IL.

\section{Quantificação da massa óssea}

O teste do Raios X de Dupla Energia é considerado como teste padrão ouro para a mensuração da $\mathrm{DMO}^{24}$. Antes de iniciar o exame do DXA, o peso corporal e a estatura foram mensurados por uma balança mecânica (Filizola ${ }^{\circledR}$, antropométrica 31, São Paulo, BRA) e por um estadiômetro profissional de parede (Physical, Terrazul ${ }^{\circledR}$, São Paulo, BRA) respectivamente.

A Densidade Mineral Óssea foi determinada por meio de um scanner da marca Hologic QDR 2000 (Hologic Inc, Walrtham, MA ${ }^{\circledR}$ ). Esse teste foi realizado com as participantes em decúbito dorsal sob uma maca acoplada ao equipamento. Em seguida, uma haste movia-se por cima da participante, emitindo raios - $X$, iniciando da cabeça em direção aos pés ${ }^{25}$. A partir da resistência encontrada pelos raios - $X$ foi estimada a DMO. Todos os testes foram feitos por uma técnica especializada no procedimento. Os locais de análise de massa óssea foram Colo do Fêmur (CF), Fêmur Total (FT) e corpo inteiro. Os indivíduos com valores de escore $T$ de massa óssea entre -1 e-2,5 DP abaixo da M foram classificados como apresentando osteopenia, enquanto indivíduos com valores abaixo de $-2,5$ foram considerados como apresentando osteoporose ${ }^{24}$.

\section{Registros alimentares}

As mulheres foram orientadas a preencher três autorregistros alimentares (horário, modo de preparo e quantidade dos alimentos consumidos em cada refeição), de modo que pelo menos um correspondesse a um dia de final de semana. Essas informações foram utilizadas para estimar o cálcio ingerido ${ }^{26}$. A média de cálcio ingerido foi expressa em miligrama por dia $\left(\mathrm{mg} / \mathrm{dia}^{-1}\right)$. A análise dos autorregistros alimentares foi realizada por uma nutricionista, por meio do software 3.0.9 Avanutri ${ }^{\circledR}$ (Avanutri informática Ltda, Rio de Janeiro, BR).

\section{Análise estatística}

As variáveis quantitativas foram descritas através de medidas de tendência central M e DP. A homogeneidade da variância entre os grupos foi testada pelo teste de Levene. A normalidade da distribuição dos dados foi verificada através do teste de Kolmogorov-Smirnov. Para a comparação das variáveis dependentes (DMO e ingestão de cálcio) entre os grupos, utilizou-se o teste $t$ de Student para amostras independentes. Todos os testes estatísticos foram realizados através do software SPSS (version 13.0, Chicago, USA), adotando-se para todos os tratamentos um nível de significância igual ou inferior a 5\% $(p<0,05)$. O cálculo do Z e T scores foi realizado de acordo com valores obtidos segundo dados da Organização Mundial de Saúde (OMS). A pontuação Z oferece uma comparação com indivíduos saudáveis da mesma idade, enquanto o escore T fornece uma comparação com adultos jovens saudáveis pareados para a mesma etnia ${ }^{24}$, ambos realizados de acordo com valores obtidos da OMS. 


\section{RES U LTA DOS}

As mulheres locadas no Grupo Intolerante a Lactose apresentaram idade, peso e estatura similares aos do GC (Tabela 1), mas estas últimas apresentaram resultados negativos para 0 diagnóstico de IL, assim como não relataram desconforto ou qualquer outro sintoma durante ou após o teste de IL. Entretanto, das 30 mulheres do GIL, 29 declararam, após o teste de IL, episódios de diarreia (96,6\%), 15 de constipação (50,0\%), 21 de náuseas $(70,0 \%), 30$ de dor e distensão abdominal $(100,0 \%)$, seis de enxaqueca $(20,0 \%)$ e 30 de flatulência (100,0\%). Pelo menos $95,5 \%$ das mulheres da amostra apresentaram mais que um desses sintomas após o teste, e 98,0\% apresentaram rejeição à ingestão de alimentos ricos em lactose.

\section{Densidade mineral óssea}

A Densidade Mineral Óssea do CF e FT do GIL foi significativamente mais baixa do que a do GC: $p<0,01$ e 0,05, respectivamente. Contudo, a DMO do corpo todo não foi diferente entre os grupos $(p>0,05)$ (Tabela 2).

O Z-escore não foi diferente entre GC e GIL para o corpo total $(M=0,43, D P=1,04$ vs $M=-0,11, D P=1,09$, respectivamente, $p>0,05)$. Entretanto, o Z-escore do CF e do FT foi significativamente mais baixo em GIL do que em GC ( $M=-0,17, D P=1,11$ e $M=-0,65, D P=1,06$ vs $M=0,43$, $D P=1,04$ e $M=0,14, D P=1,25$, respectivamente, $p<0,05)$. De acordo com o escore T, no GC, $60 \%$

Tabela 2. Densidade mineral óssea nos diferentes sítios para o grupo-controle e para o grupo intolerante à lactose. Maceió (AL), 2011.

\begin{tabular}{lcclllll}
\hline \multirow{2}{*}{ Variável } & \multicolumn{2}{c}{$\mathrm{GC}$} & & \multicolumn{2}{c}{ GIL } & \multirow{2}{*}{$p$} \\
\cline { 2 - 3 } & $\mathrm{M}$ & $\mathrm{DP}$ & & $\mathrm{M}$ & $\mathrm{DP}$ & \\
\hline DMO CF $\left(\mathrm{g} / \mathrm{cm}^{2}\right)$ & 0,86 & 0,13 & & 0,77 & 0,12 & $0,01^{*}$ \\
DMO FT $\left(\mathrm{g} / \mathrm{cm}^{2}\right)$ & 1,14 & 0,14 & & 1,06 & 0,12 & $0,01^{*}$ \\
Corpo todo $\left(\mathrm{g} / \mathrm{cm}^{2}\right)$ & 1,14 & 0,15 & & 1,08 & 0,09 & 0,09 \\
\hline
\end{tabular}

"Significativamente diferente entre os dois grupos. GC: Grupo-Controle; GIL: Grupo Intolerante à Lactose; DMO: Densidade Mineral Ossea; CF: Colo de Fêmur; FT: Fêmur Total; M: Média; DP: Desvio-Padrão. das mulheres foram classificadas como tendo massa óssea normal, 37\% como tendo osteopenia e 3\% tendo osteoporose. No GIL, 43\% apresentaram massa óssea normal, $56 \%$ osteopenia e $3 \%$ osteoporose.

\section{Ingestão de cálcio}

O consumo de cálcio foi menor $(p<0,001)$ no GIL ( $\left.M=250,5, D P=111,7 \mathrm{mg} / \mathrm{dia}^{-1}\right)$ do que no GC (M=659,7, DP=316,1 $\left.\mathrm{mg} / \mathrm{dia}^{-1}\right)$.

\section{DISCUSS Ã O}

Os principais achados do presente estudo foram: 1) redução significativa no consumo de cálcio no GIL; 2) diminuição na DMO tanto do CF quanto do FT no GIL.

Apesar dos resultados relevantes, o presente estudo apresenta algumas limitações que precisam ser mencionadas. A não utilização do teste de hidrogênio expirado para o diagnóstico da má-absorção da lactose talvez seja uma delas, uma vez que esse teste é um dos mais utilizados e precisos para o diagnóstico de má-absorção. Entretanto, o teste utilizado no presente estudo apresenta um alto grau de validade relatado na literatura ${ }^{22,23}$, o que sugere que esse fator pode ter interferido minimamente nos resultados encontrados.

No presente estudo, verificou-se redução significativa no consumo de cálcio no GIL quando comparada à do grupo-controle $(M=250,5$, $\mathrm{DP}=111,7$ vs $\left.\mathrm{M}=659,7, \mathrm{DP}=316,1 \mathrm{mg} / \mathrm{dia}^{-1}\right)$. Esses resultados estão de acordo com outros estudos que encontraram baixo consumo de cálcio para indivíduos com IL, com valores entre $400 \mathrm{mg} / \mathrm{dia}^{-1}$ a $685 \mathrm{mg} / \mathrm{dia}^{-1}$ para o grupo IL, e de $800 \mathrm{mg} / \mathrm{dia}^{-1}$ a $905 \mathrm{mg} / \mathrm{dia}^{-1}$ para o grupo-controle $\mathrm{e}^{9,12,13,19,27}$. Entretanto, os valores de ingestão de cálcio em ambos os grupos (controle e intolerante) do presente estudo são consideravelmente menores do que os relatados na literatura9,12,13,19,27. Uma possível explicação para essa variação do baixo consumo de cálcio por indivíduos adultos saudá- 
veis seria uma possível diferença cultural e de hábitos alimentares das mulheres do presente estudo em relação às recrutadas em outros estudos na literatura.

Realmente, alguns estudos sugerem consumo inadequado e abaixo do recomendado de cálcio em algumas populações ${ }^{15,12,27}$. Contudo, as diferenças no consumo de cálcio entre indivíduos com IL e controle relatados na literatura ${ }^{9,12,13,19,27}$ é de aproximadamente $285 \mathrm{mg} / \mathrm{dia}^{-1}$ a $400 \mathrm{mg} / \mathrm{dia}^{-1}$, muito similar à diferença encontrada no presente estudo $\left(\sim 400 \mathrm{mg} / \mathrm{dia}^{-1}\right)$. Assim, os resultados encontrados aqui reforçam a ideia de um menor consumo de cálcio por mulheres IL na idade adulta. Provavelmente, esse baixo consumo de cálcio do GIL em relação aos dados reportados na literatura seja um reflexo dos inúmeros e severos sintomas de intolerância ao leite apresentados por essas mulheres, causando rejeição diária ao seu consumo devido ao aparecimento de sintomas gastrointestinais e ao mal-estar apresentados após a ingestão desses alimentos ${ }^{14,28}$.

De fato, alguns estudos mostraram que a rejeição pode ser também uma das causas para o baixo consumo de cálcio em indivíduos com IL 14,28. Quando a absorção de cálcio pela dieta é insuficiente para repor as perdas diárias, o cálcio é retirado dos ossos, contribuindo para um balanço negativo no equilíbrio da remodelação óssea. Nesse sentido, alguns estudos encontraram diferenças significativas na DMO do CF entre o grupo-controle e o grupo com IL 12,14,15,19,21.

No presente estudo, foram encontradas diferenças entre os grupos para a DMO do CF e FT de $-10,5 \%$ e $-6,5 \%$ respectivamente. Provavelmente, à medida que a DMO diminui, observa-se um aumento do risco de fratura independente da idade ${ }^{28}$. Ao ser avaliada a prevalência de osteoporose e de osteopenia, observou-se que o grupo com IL apresentou $57,0 \%$ de casos de osteopenia e 3,0\% de osteoporose, o que aumenta consideravelmente o risco de fraturas nesse grupo. Resultado similar foi encontrado por Di
Stefanno et al. ${ }^{19}: 41,0 \%$ de osteopenia no CF nos indivíduos GIL. Assim, apesar da possibilidade de existir outros fatores que poderiam afetar a DMO, tem-se o reduzido consumo de cálcio no GIL - muito abaixo dos $1.000 \mathrm{mg} / \mathrm{dia}^{-1}$ recomendados $^{29}{ }^{-}$, um dos fatores determinantes para esse quadro. A baixa ingestão de cálcio se deve, provavelmente, a uma baixa ingestão de leite, queijo, iogurte e outros derivados em mulheres IL, conforme descrito na literatura15,17,27,28. Assim, devido ao baixo consumo de leite e derivados, é esperado que exista um balanço negativo de cálcio e que se instale um quadro de fragilidade óssea compatível com osteopenia/osteoporose.

\section{CON CLUSÃO}

Os resultados obtidos no presente estudo sugerem que mulheres adultas com IL apresentem um baixo consumo de cálcio dietético quando comparadas a mulheres saudáveis, provavelmente devido ao reduzido consumo de leite e derivados motivado pelos sintomas de IL. O baixo consumo de cálcio provavelmente levou a uma redução da DMO no CF e no FT nos indivíduos com IL, predispondo à osteopenia e à osteoporose.

\section{A GRADECIMENTOS}

A equipe do Laboratório de Análises Clínicas de Alagoas, a equipe da Clínica Sonograf e a nutricionista Fabiana Palmeira pelo auxílio na análise dos autorregistros alimentares.

\section{COLABORADORES}

NA SALOMÃO concebeu a ideia do estudo, realizou o levantamento bibliográfico, coletou os dados e escreveu o trabalho. TA SILVA realizou a análise dos dados, contribuiu com a discussão dos resultados e escreveu parte do trabalho. AAR GERALDES e AE LIMA-SILVA auxiliaram na elaboração da ideia, no desenho experimental do estudo, na interpretação dos dados e na revisão do trabalho. 


\section{REFERÊ NCIAS}

1. Guéguen L, Pointillart A. The bioavailability of dietary calcium. J Am Coll Nutr. 2000; 19(2 Suppl): 119S-36S.

2. Roberson CM. Lactose intolerance. Ala Nurse. 2004; 31(4):23-4.

3. Uggini PL, Fagundes RLM. Tratamento dietético da intolerância à lactose infantil: teor de lactose em alimentos. Hig Aliment. 2006; 21(140):24-9.

4. Hovde O, Farup PG. A comparison of diagnostic tests of lactose malabsorption: which one is the best? BMC Gastroenterol. 2009; 9:82. doi: 10.11 86/1471-230X-9-82.

5. Robayo-Torres CC, Nichols BL. Molecular differentiation of congenital lactase deficiency from adult-type hypolactasia. Nutr Rev. 2007; 65(2):95-8. doi: 10.1 111/j.1753-4887.2007.tb00286.x.

6. Sibley E. Carbohydrate intolerance. Curr Opin Gastroenterol. 2004; 20(2):162-7.

7. Ingram CJ, Raga TO, Tarekegn A, Browning SL, Elamin MF, Bekele $\mathrm{E}$, et al. Multiple rare variants as a cause of a common phenotype: several different lactase persistence associated allelesin a single ethnic group. J Mol Evol. 2009; 69(6):579-88. doi: 10.1007/s00239-009-9301-y.

8. Di Stefano M, Terulla V, Tana P, Mazzocchi S, Romero E, Corazza GR. Genetic test for lactase non-persistence and hydrogen breath test: is genotype better than phenotype to diagnose lactose malabsorption? Dig Liver Dis. 2009; 41(7):474-9. doi: 10.1016/j.dld.2008.09.020.

9. Brannon PM, Carpenter TO, Fernandez JR, Gilsanz V, Gould JB, Hall KE, et al. NIH Consensus Development Conference Statement: lactose intolerance and health. NIH Consens State Sci Statements. 2010; 27(2): Epub ahead of print.

10. Savaiano D. Lactose intolerance: an unnecessary risk for low bone density. Nestle Nutr Workshop Ser Pediatr Program. 2011; 67:161-71. doi: 10.11 59/000325582.

11. Montalto M, Gallo A. Sufficient evidence that $12 \mathrm{~g}$ of lactose is tolerated by most adults with lactose malabsorption and intolerance but insufficient evidence on the effectiveness of therapeutical strategies tested so far. Evid Based Med. 2010; 15(6):172-3. doi: 10.1136/ebm1130.

12. Honkanen R, Kröger $H$, Alhava $E$, Turpeinen $P$, Tuppurainen M, Saarikoski S. Lactose intolerance associated with fractures of weight-gearing bones in finish women aged 38-57 years. Bone. 1997; 21(6): 473-7. doi: 10.1016/58756-3282/97/00172-5.
13. Kudlacek S, Freudenthaler O, Weissböerck H, Schneider B, Willvonseder R. Lactose intolerance: a risk factor for reduced bone mineral density and vertebral fractures? J Gastroenterol. 2002; 37(2): 1014-9. doi: 10.1007/s005350200171.

14. Obemayer-Pietsch MB, Gugatschka M, Reitter S, Plank W, Strele A, Walter D, et al. Adult-type hypolactasia and calcium availability: decreased calcium intake or impaired calcium absorption? Osteoporos Int. 2007; 18(4):445-51. doi: 10.1007/ s00198-006-0251-6.

15. Corazza GR, Benati G, Di Sario A, Tarozzi C, Strocchi $A$, Passeri $M$, et al. Lactose intolerance and bone mass in postmenopausal Italian women. $\mathrm{Br} J$ Nutr. 1995; 73(3): 479-87. doi: 10.1079/BJN19950050.

16. Matlik L, Savaiano D, McCabe G, Vanloan M, Blue $\mathrm{CL}$, Boushey CJ. Perceived milk intolerance is related to bone mineral content in 10- to 13-year-old female adolescents. Pediatrics. 2007; 120(3): e669-e77. doi: 10.1542/peds.2006-1240.

17. Carroccio A, Montalto G, Cavera G, Notarbatolo AA. Lactose intolerance and self-reported Milk intolerance: relationship with lactose maldigestion and nutrient intake. J Am Coll Nutr. 1998; 17(6): 631-6.

18. Aloha H. Diagnosis of hypolactasia and lactose malabsorpition. Scand J Gastroenterol Suppl. 1994; 202:26-35.

19. Di Stefano M, Veneto G, Malservisi S, Cecchett L, Minguzzi L, Strocchi A, et al. Lactose malabsorption and intolerance and peak bone mass. Gastroenterology. 2002; 122(7):1793-9. doi: 10.10 53/gast.2002.33600.

20. Ulrich CM, Georgiou CC, Snow-Harter CM, Gillis $\mathrm{DE}$. Bone mineral density in mother-daughter pairs: relations to lifetime exercise, lifetime milk consumption, and calcium supplements. Am J Clin Nutr. 1996; 63(1):72-9.

21. Nieves JW, Komar L, Cosman F, Lindsay R. Calcium potentiates the effect of estrogen and calcitonin on bone mass: review and analysis. Am J Clin Nutr. 1998; 67(1):18-24.

22. Séva-Pereira A. Malabsorção de lactose do adulto em uma população brasileira [doutorado]. Campinas: Universidade Estadual de Campinas; 1981.

23. Isokoski M, Jussila J, Sarna S. A simple screening method for lactose malabsorption. Gastroenterology. 1972; 62(1):28-32.

24. World Health Organization. Assessment of fracture risk and its application to screening for postmenopausal osteoporosis. Report of a WHO 
Study Group. World Health Organ Tech Rep Ser. 1994; 843:1-129.

25. Fornetti WC, Pivarnik JM, Foley JM, Fiechtner JJ. Reliability and validity of body composition measures in female athletes. J Appl Physiol. 1999; 87(3):1114-22.

26. Crawford PB, Wang MC, Sabry ZI, Hudes M, VanLoan M, Davis D, et al. Adolescent diet is predictive of peak bone mass. Am J Clin Nutr. 2002; 75:356s.

27. Ennatah N, Pekkarien T, Välimäki MJ, Löyttyniemi E, Järvela I. Genetically defined adult-type hypolactasia and self-report lactose intolerance as risk factors of osteoporosis in finish postmeno- pausal women. Eur J Clin Nutr. 2005; 59(10): 1105-11. doi:10.1038/sj.ejcn.1602219.

28. Enattah NS, Kuokkanen M, Forsblom C, Natah S, Oksanen A, Jarvela I, et al. Correlation of intestinal disaccharidase activities with the C/T-13910 variant and age. World J Gastroenterol. 2007; 13(25): 3508-12.

29. Institute of Medicine. Dietary reference intakes: the essential guide to nutrient requirements. Washington (DC): National Academy Press; 2006.

Recebido em: 8/2/2012

Versão final em: $4 / 6 / 2012$

Aprovado em: 25/6/2012 
